\title{
The Design of Shell-and-tube Heat Exchanger in the Project of the Coal Bed Methane Electrical Power Generation
}

\author{
Lan Xiangyun ${ }^{1,2}$ \\ ${ }^{1}$ State Key Laboratory of Gas Desaster Monitoring and Emergency Technology, Chongqing China \\ ${ }^{2}$ China Coal Technology and Engineering Group Chongqing Rrsearch Institute, Chongqing, China
}

Email address:

lanxiangyun $@$ cqccteg.com

\section{To cite this article:}

Lan Xiangyun. The Design of Shell-and-tube Heat Exchanger in the Project of the Coal Bed Methane Electrical Power Generation. Journal of Chemical, Environmental and Biological Engineering. Vol. 4, No. 2, 2020, pp. 53-59. doi: 10.11648/j.jcebe.20200402.14

Received: April 24, 2020; Accepted: May 18, 2020; Published: July 23, 2020

\begin{abstract}
Coal-bed-methane (CBD) electrical power generation is an active and effective measure to reduce emissions of greenhouse gases. Using shell-and-tube heat exchangers to reduce the water content of pipeline gas can improve the efficiency of the generator set. Designing heat exchanger by ASPEN EDR software can save a lot of manual calculation process, so as to improve the efficiency of the heat exchanger designer. The general method of designing and verifying shell-and-tube heat exchanger by EDR ASPEN software is described in this article by the example of the local process of low concentration gas power generation project. Because of the heat flux medium is low concentration coal bed methane with high explosion risk, anti-explosion measures must be taken to ensure safety beyond the requirements of normal heat exchangers of heat transfer, pressure drop.
\end{abstract}

Keywords: Shell-and-Tube Heat Exchanger, ASPEN EDR, Coal Bed Methane Electrical Power Generation, Gas Utilization, Greenhouse Gas

\section{Introduction}

The technology of coal bed methane (CBD) electrical power generation is a very effective energy saving and emission reduction measure, which can not only reduce the emissions of greenhouse gases, but also produce electrical energy to produce economic benefits. In low-concentration methane $\left(\mathrm{CH}_{4} \approx 10 \%\right)$ electrical generationg projects, the moisture in the CBD will affect the performance of the generator. The high-temperature waste heat generated by the generator can be used to generate low-temperature refrigerants through the lithium bromide absorption refrigerator [1], refrigerants and low-concentration coal seams. The raw material gas is heat-exchanged in a shell-and-tube heat exchanger, the temperature of the raw material gas is reduced, and part of the water is condensed from the raw material gas. Reducing the water content of the CBD can improve the working efficiency and stability of the low-concentration coal bed methane generator. In this technology, the design of the shell and tube heat exchanger is the key work.

Heat exchanger is a device that transfers heat between two or more fluids, and is widely used in chemical, petroleum, power, food and other industries. The heat exchanger can be used as a heater, cooler, condenser, evaporator and reboiler, etc. according to the actual working requirements, and it is widely used. In chemical companies, heat exchanger investment generally accounts for $10 \%-20 \%$; in oil refineries, heat exchanger investment accounts for about $35 \%-40 \%$ of the total investment [2-5]. There are many types of heat exchangers. Tube and shell heat exchangers are the most widely used in various types of heat exchangers because of their low manufacturing cost, easy cleaning, large processing capacity, stable and reliable operation, and large operating temperature and pressure range. According to its structural characteristics, the shell and tube heat exchanger can be divided into fixed tube plate type, floating head type, U-shaped tube type, etc. Generally, the tube and shell type heat exchanger is selected according to the specific conditions of fluid type, temperature range, operating pressure, and the type of device [6].

This article takes this shell-and-tube heat exchanger as an example to describe the general method of designing a shell-and-tube heat exchanger using the first block (Shell \& 
Tube Exchanger) of ASPEN EDR software. Since the heat flow medium is $10 \%$ concentration oxygenated coal bed methane, the heat exchanger will be corroded due to the presence of oxygen. And the concentration of coal bed methane is within the explosion limit range [7-8], so explosion-proof or explosion-proof measures must be taken in the design of the heat exchanger.

\section{Design of Shell and Tube Heat Exchanger}

Advanced System for Process Engineering (ASPEN) chemical simulation system is large-scale chemical simulation software developed by the famous Massachusetts Institute of Technology (MIT) in the 1970s. In order to commercialize it, ASPEN Tech was established in 1982 and called it ASPEN Plus. After decades of development, ASPEN Plus process simulation software has been widely used in chemical, energy, environmental protection and other industries [9]. At present, ASPEN Plus has become one of the most popular process simulation software in the world [10]. Exchanger Design and Rating (EDR) is a special module for heat exchangers in the ASPEN Plus software system. Engineers or researchers can use the EDR module to easily design and verify heat exchangers. EDR software can perform heat exchange calculation based on the input parameters, which eliminating a lot of manual calculation processes and greatly improving the efficiency of the heat exchanger design.

The ASPEN Plus EDR module (hereinafter referred to as EDR software) has the following heat exchanger blocks:

(i) ASPEN Shell \& Tube Exchanger, which is used for simulation design and verification of various shell and tube heat exchangers;

(ii) ASPEN Shell \& Tube Mechanical, which is used for the mechanical design or rating of shell and tube heat exchangers and basic pressure vessels;

(iii) ASPEN Plate Exchanger, which is used for simulation design and verification of plate heat exchanger;

(iv) ASPEN Air Cooled Exchanger, which is used for simulation design and verification of air cooler;

(v) ASPEN Fired Heater, which is used for simulation design and verification of flame heater;

(vi) ASPEN Plate Fin Exchanger, which is used for simulation design and verification of plate-fin heat exchanger.

\subsection{Data Preparation}

Table 1 lists the inlet and outlet parameters of the cold and heat flow (refrigerant and raw material gas) in the shell and tube heat exchanger. It is worth noting that when providing the inlet and outlet parameters of the heat exchanger, it must be ensured that the heat exchanger can be calculated. The load and complete import and export parameters are the prerequisites for designing shell and tube heat exchangers using EDR.
Table 1. In/out parameter of hot and cold fluid of shell-and-tube heat exchanger.

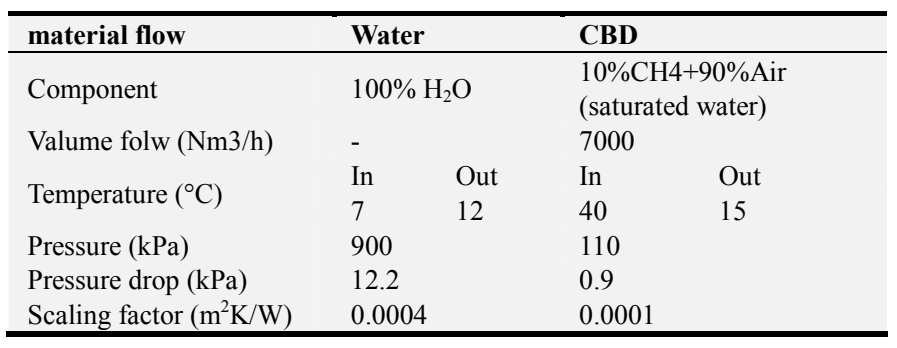

After checking the "Saturated Vapor Pressure Table of Water", the saturated vapor pressure of water at $40^{\circ} \mathrm{C}$ is $7.3814 \mathrm{KPa}$. According to the law of partial pressure of gas, combined with the ideal gas state equation, the ratio of nitrogen and oxygen in the air is calculated according to 79:21. Available:

$$
\begin{gathered}
n a: n b: n c: n d=7.3814:(110-7.3184) \times 10 \%:(110-7.3184) \\
\times 90 \% \times 79 \%:(110-7.3184) \times 90 \% \times 21 \% \\
\quad=7.3184: 10.2682: 73.0066: 19.4086 \\
\quad=0.0665: 0.0933: 0.6637: 0.1764
\end{gathered}
$$

In formula (1), $n_{\mathrm{a}}-n_{\mathrm{d}}$ are the mole percentages of water vapor, methane, nitrogen, and oxygen, respectively. Relative molecular mass of raw gas (mixture) available

$$
\begin{array}{r}
M=18 n_{a}+16 n_{b}+28 n_{c}+32 n_{d}= \\
(18 \times 0.0665+16 \times 0.0933+28 \times 0.6637+32 \times 0.1764) \mathrm{g} / \mathrm{mol} \\
=26.9182 \mathrm{~g} / \mathrm{mol}
\end{array}
$$

Since the low concentration coalbed methane is low temperature and low pressure, the ideal gas state equation and density equation can be selected:

$$
\begin{gathered}
P V=n R T=\frac{m}{M} R T \\
\rho=\frac{m}{V}
\end{gathered}
$$

Where, $\mathrm{P}$ is the pressure, $\mathrm{kPa} ; \mathrm{V}$ is the volume, $\mathrm{m} 3 ; n$ is the amount of substance, mol; $\mathrm{R}$ is the gas universal constant, $8.314510 \mathrm{~J} /(\mathrm{mol} \bullet \mathrm{K}) ; \mathrm{m}$ is the mass, $\mathrm{kg}$; Relative molecular mass, $\mathrm{g} / \mathrm{mol}$; $\mathrm{T}$ is temperature, $\mathrm{K} ; \rho$ is density, $\mathrm{kg} / \mathrm{m}^{3}$.

Combining formula (2), formula (3), we get:

$$
\rho=\frac{M P}{R T}
$$

Substitute the data to calculate:

$$
\rho=\frac{26.9182 \mathrm{~g} / \mathrm{mol} \times 110 \mathrm{KPa}}{8.3145 \mathrm{~J} / \mathrm{mol} \cdot \mathrm{K} \times 313.15 \mathrm{~K}}=1.1372 \mathrm{~kg} / \mathrm{m}^{3}
$$

Therefore, the feed gas mass flow:

$$
\mathrm{Q}=1.1372 \mathrm{~kg} / \mathrm{m}^{3} \times 7000 \mathrm{~m}^{3} / \mathrm{h}=7960 \mathrm{~kg} / \mathrm{h}
$$




\subsection{Choice of Runner}

Under the Application Options tab of the EDR main interface, you can define the "design" or "check" mode of the heat exchanger. When you first design the shell and tube heat exchanger, select "Design", which is the design mode. After selecting the design mode, the next step is to define the hot runner. When choosing a logistics channel, it is mainly based on the following principles [11]:

(i) Temperature. High-temperature fluids generally go through the tube, because high temperature will reduce the allowable stress of the material, high-temperature fluids can save insulation and reduce the thickness of the shell. Sometimes, in order to facilitate the heat dissipation of high-temperature fluids, the high-temperature fluids can also be taken to the shell side

(ii) Pressure. Fluids with too high pressure should take the tube path, which can reduce the thickness of the shell and save costs.

(iii) Cleanliness. Easy-to-clean fluids are easy to clean due to easy-to-scale fluids. (iv) Coefficient of heat transfer film. Fluids with small heat transfer film coefficients should take the tube path, which is conducive to heat exchange.

(v) Corrosive. Highly corrosive fluids take the tube path, saving corrosion-resistant materials.

Because the heat flow medium is $10 \%$ concentration CBM, the heat exchanger will be corroded due to the presence of oxygen. The material used in the shell is much larger than that of the tube bundle. In order to reduce costs, the heat exchanger is designed for the raw material gas to go through the tube path. The tube material is stainless steel and the shell material is carbon steel.

\subsection{Parameter Input}

Enter Input / Problem Definition / Process Data in turn, enter the logistics parameter input interface as shown in Figure 1, first select the unit in the upper left corner of Figure 1 as the metric unit Metric, and then fill in the blanks in sequence according to the data in Table 1 and the calculated results Place, as shown in Figure 1

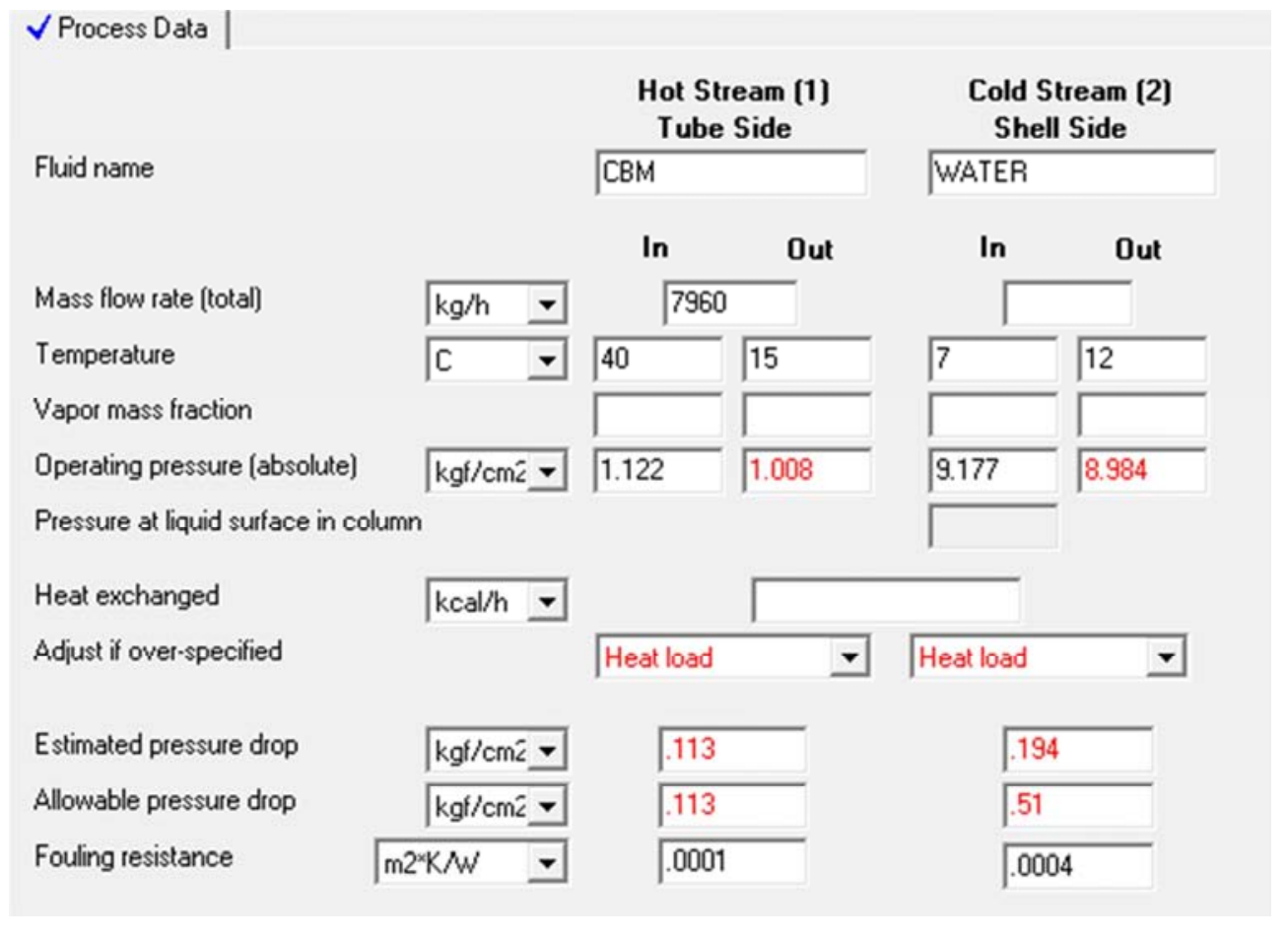

Figure 1. Input interface of fluid parameter.

In the left navigation bar of the EDR software window, select Shell \& Tube / Property Date / Hot Steam Composition, select the B-JAC data package at the Physical property package, and select the mole percentage in the next line. Fill in the blanks.

For the CBM with a heat flow of $40^{\circ} \mathrm{C}$ and an absolute pressure of $110 \mathrm{KPa}$, directly select the ideal gas state equation in the Property Methods tab at Figure 2. After selecting the physical property equation, select Hot Stream Properties from the navigation bar on the left, and click Get Properties in the upper left corner of the pop-up window to see the physical property parameters of the heat flow.
According to the same method, the components of the cold flow and the physical property equation can be obtained, and the physical property parameters of the cold flow can be obtained.

\subsection{Heat Transfer Calculation}

After the cold and hot flow import and export parameters and physical properties parameters are prepared, click the run button in the EDR software function area. The software will pop up a new window to display a series of calculation processes. Click on the left navigation bar of the EDR 
software and select Results / Result Summary / TEMA Sheet in sequence. You can see the preliminary design results of the heat exchanger from the TEMA data sheet (Figure 2). This design result is not the final heat exchanger parameter, but the basic data support for the subsequent heat exchanger verification.

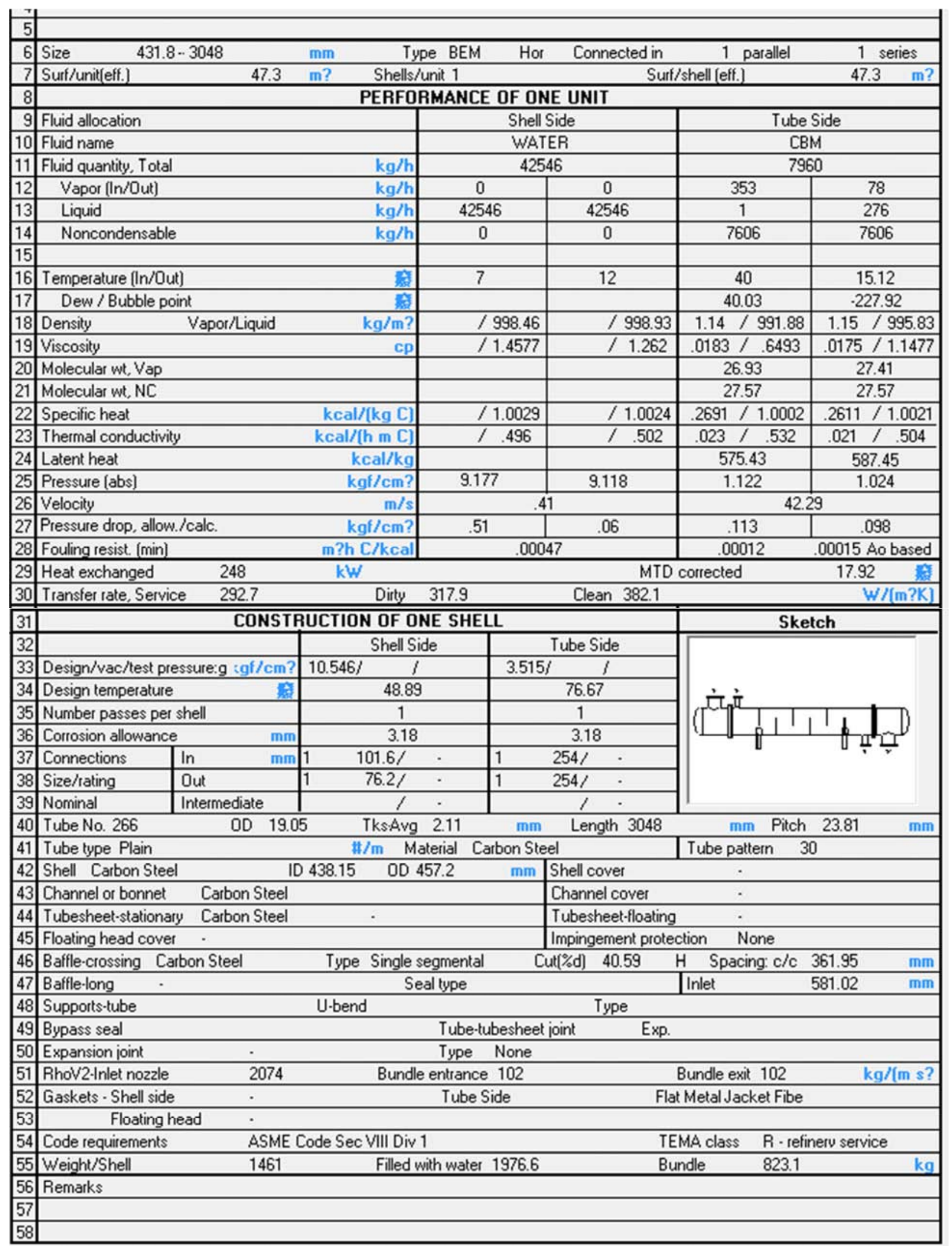

Figure 2. The result of TEMA sheet. 


\section{Checking the Heat Exchanger}

\subsection{Structural Parameters of the Heat Exchanger}

After the preliminary design of the heat exchanger is completed, select Problem Definition / Application Options and select Rating / Checking in Calculation mode. You can see that there are several small red crosses on the left to enter the heat exchanger "check" mode.

The blue part under the interface of these small red crosses indicates what we need to improve (Figure 3), mainly including the basic parameters of the shell and tube heat exchanger type, shell, tube bundle, baffle spacing and other parameters. After setting these parameters, the red cross will disappear, click the run button, the heat exchanger will automatically calculate and get the TEMA table. What we need to do next is to repeat the process of "modifying the parameters-calculating the TEMA table-verifying whether the heat transfer requirements and pressure drop requirements are met" until the calculation results meet the heat transfer requirements and pressure drop requirements of Figure 1.

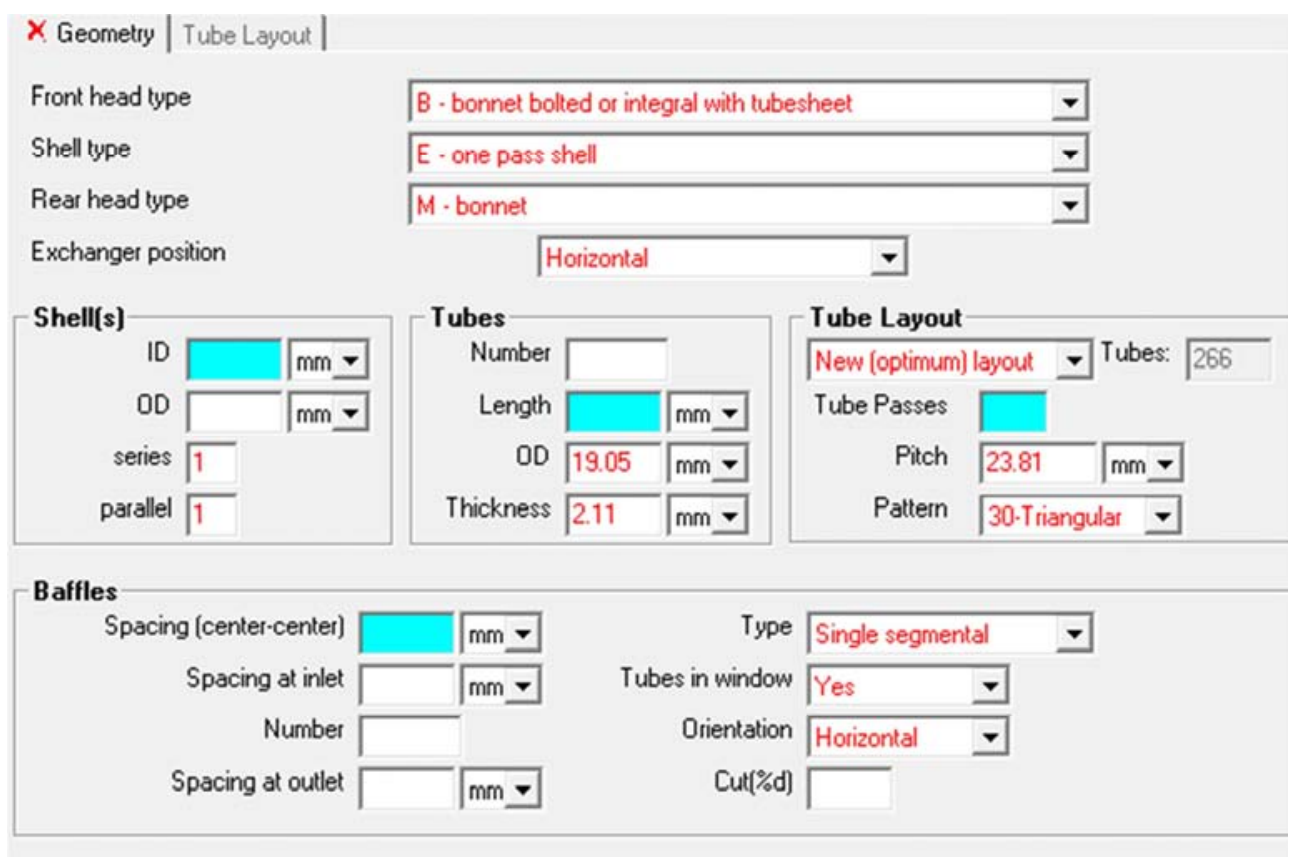

Figure 3. The interface of structural design of heat exchanger.

The shell and tube heat exchanger type is the most commonly used fixed tube plate heat exchanger (BEM). The center distance of the baffle plate is $300 \mathrm{~mm}$. Since the pressure drop in the tube pass required by Table 1 is less than $0.9 \mathrm{kPa}$, the heat exchanger is set to a single tube pass. The three important parameters of the shell inner diameter, tube length, and tube diameter are gradually modified according to the results of the preliminary design of the heat exchanger (Figure 2). The main relationships are as follows:

(i) The flow velocity is negatively related to the pipe diameter and the number of one-way pipes;

(ii) The heat transfer coefficient is positively related to the fluid flow rate;

(iii) The pressure drop is positively correlated with the flow rate and positively correlated with the tube length;

(iv) The pipe diameter and the number of one-way pipes are positively related to the inner diameter of the shell.

First analyze the TEMA table shown in Figure 2: The gas velocity of the tube side gas is $42.29 \mathrm{~m} / \mathrm{s}$, far exceeding the general velocity of the tube side gas; the tube side pressure drop is $9.577 \mathrm{kPa}$, which is greater than the pressure drop required by Table 1 by $0.9 \mathrm{kPa}$ Therefore, the first thing to consider is to increase the tube bundle and increase the tube diameter to reduce the gas flow rate in the tube side. As the flow rate decreases and the heat transfer coefficient decreases, the tube length must be increased to meet the heat exchange requirements. After adjusting the pipe diameter, pipe number and pipe length many times, the final diameter is $35 \mathrm{~mm}$, the number of pipes is 350 , and the pipe length is $4.5 \mathrm{~m}$. The calculation result of Figure 4 can be obtained.

\subsection{Check of TEMA Data Sheet}

Open the TEMA data sheet from Results / Result Summary / TEMA Sheet and check whether the heat exchanger meets the design conditions. Part of the TEMA data table is shown in Figure 4. The data in line 26 shows that the flow rate of the cooling water in the shell side is $0.46 \mathrm{~m} / \mathrm{s}$, and the flow rate of the coalbed methane in the tube side fluid is $7.46 \mathrm{~m} / \mathrm{s}$. From the data in line 27 , it can be seen that the shell-side pressure drop is $7.974 \mathrm{kPa}$, and the tube-side pressure drop is $0.877 \mathrm{kPa}$, all of which meet the pressure drop requirements of Table 1 . The content of line 30 is the heat exchange coefficient required by the heat exchanger is $79.7 \mathrm{~W} / \mathrm{m} \cdot \mathrm{K}$, the heat exchange coefficient under the fouling coefficient is $101.3 \mathrm{~W} / \mathrm{m} \cdot \mathrm{K}$, and the affluence coefficient is 1.27 to meet the heat exchange 
requirements.

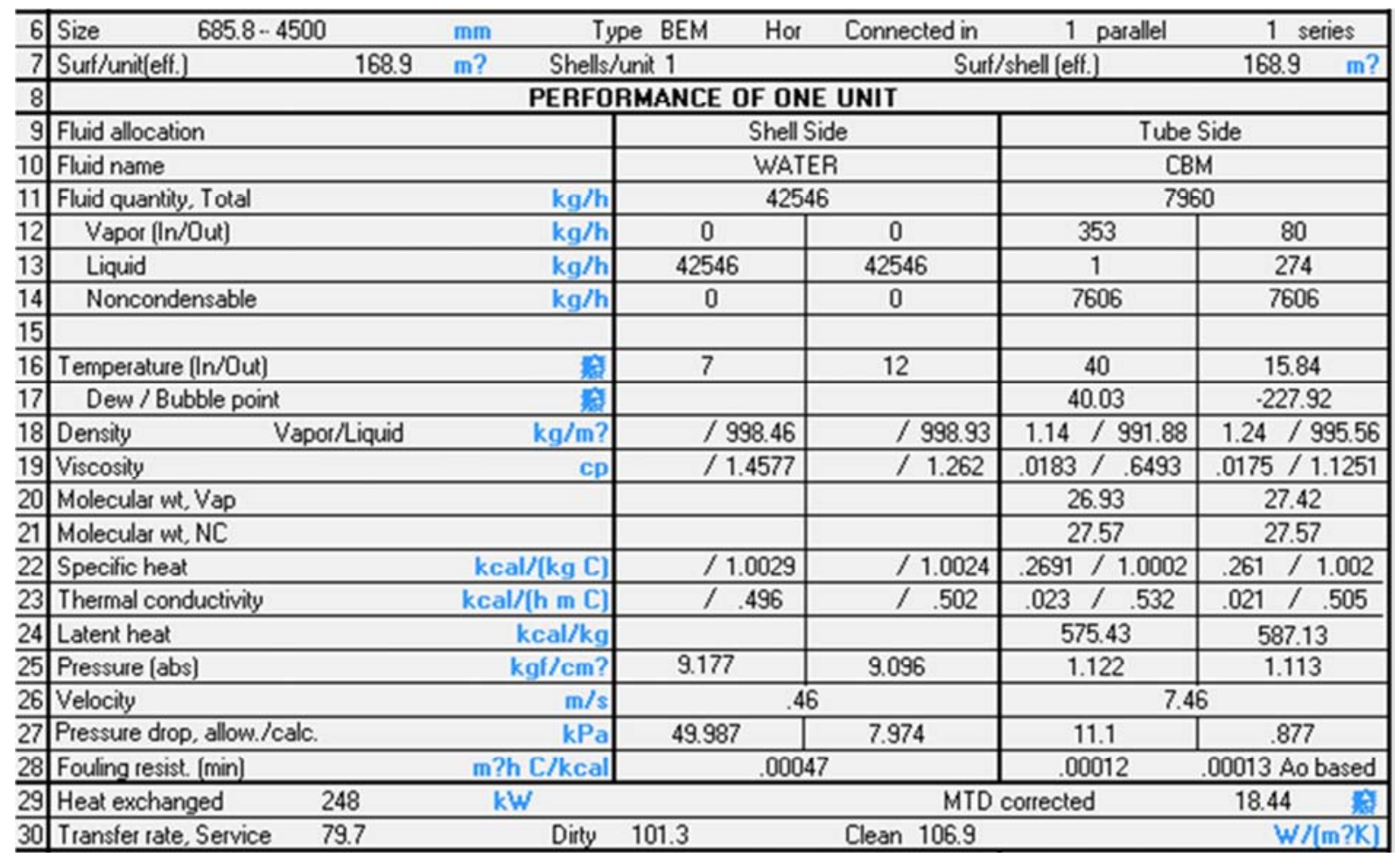

Figure 4. Part of TEMA sheet.

In addition to these most important parameters, the navigation bar Input / Exchanger Geometry and other sub-directories can also design specific parameters of nozzles, tube arrangement, baffles, fins and other structures. In general, EDR software will have a default choice. For some special heat exchangers, the designer will design the parameters according to the specific needs. Every time you modify a structural parameter, click Run to get a new calculation result, saving a lot of manual calculation.

In the heat exchanger verification stage, the designer can modify all structural parameters of the heat exchanger under the Input / Exchanger Geometry catalog. Therefore, the designer's process of modifying these parameters and comparing the calculation results many times is the process of optimizing the heat exchanger. Through optimization of the heat exchanger, the designer finally designed an economical and practical heat exchanger.

\section{The Safety of Heat Exchanger}

Since there is no fire source inside the heat exchanger, and the maximum temperature of the medium is $40^{\circ} \mathrm{C}$, which is far lower than the ignition point of the gas, it can be considered that there is no explosion source inside the heat exchanger. However, the gas concentration of the pipeline is $10 \%$, which is within the range of gas explosion. The flame may be directly transmitted to the heat exchanger through the gas transmission pipeline.

It can be seen from the TEMA data sheet that the raw gas has $274 \mathrm{~kg} / \mathrm{h}$ of water vapor condensed. A gas-liquid separation tank with "lower inlet and upper outlet" is arranged at the outlet of the raw material gas of the heat exchanger. The inlet of the separation tank is below the liquid level, and the design pressure of the separation tank is increased to more than $1.0 \mathrm{Mpa}$, which is equivalent to a water-sealed tank [12], can effectively prevent flames and shock waves from pouring into the heat exchanger.

At the front end of the tube side entrance of the heat exchanger, a flameproof device is installed to prevent flame propagation. After decades of development, explosion suppression technology and equipment have become increasingly reliable in low-concentration coalbed methane transportation [13-15]. Before the inlet of the raw material gas of the heat exchanger, a set of explosion suppression device is installed to effectively ensure the safety of the heat exchanger.

After taking measures, the heat exchanger will not be damaged by flames and shock waves, and there is no power equipment and fire source inside the heat exchanger. It can be considered that the heat exchanger is safe and reliable.

\section{Conclusion}

This article takes the local process of the residual heat cooling of the low-concentration gas power generation project as an example to introduce the process of designing the shell-and-tube heat exchanger using the Exchanger Design and Rating (EDR) module in the ASPEN Plus software, and performs the calculation.

The general steps for designing a heat exchanger with 
EDR software are: the first step is to select the design mode, enter the inlet and outlet temperatures of the cold and hot flow, pressure parameters, enter the mass or mole percentage of each component of the cold and hot flow, and select the physical property calculation method. After completing the above steps, click Run to get the preliminary design results from the TEMA table. The second part selects the check mode and carries out the structural design of the heat exchanger: select the type of the heat exchanger, enter the diameter of the shell, the diameter and length of the heat exchange tube, the tube path, the spacing of the baffle plate, etc., run the software, from the TEMA table Observe whether the design results meet the pressure drop and heat exchange requirements of the heat exchanger. If it is not satisfied, repeat the second step and change the structural parameters until the design requirements are met.

Heat exchangers with other special requirements must be specially designed. For example, the heat flow medium of the heat exchanger is coal-bed gas within the explosion limit. In order to ensure the safety of the heat exchanger, a flameproof device must be placed at the front end of the heat exchanger and a gas-liquid separation tank at the rear end.

\section{Acknowledgements}

This study was financially supported by National Natural Science Foundation of China (51874348, 51574280), Science and Technology Innovation and Entrepreneurship Fund of China Coal Technology Engineering Group (2019-TD-QN040). General Projects of Chongqing Research Institute of China Coal Technology and Engineering Group Corporation (2019YBXM28).

\section{References}

[1] Liu, G P. (2010) Thermal Economic Analysis of Mine Cooling System for Gas Power Generation Waste Heat Refrigeration Mine [C]. Proceedings of 2010 Annual Conference of China Occupational Safety and Health Association. 346-351.

[2] Dong Q W, Zhang Y. (2008) Heat Exchanger [M]. Chemical Industry Press, Beijing.
[3] Fu L, Zeng D L, Tang K L, Jia H Y. (2012) Numerical Simulation Study of Shell-Side Fluid Flow and Heat Transfer in Shell-and-Tube Heat Exchanger [J]. Pressure Vessel Technology. 0536-41.

[4] Qi H Y, Gao L, Zhang Y Y, Zhou C L. (2012) Overview of the Shell and Tube Heat Exchangers about Heat Transfer Enhancement Technology [J]. Pressure Vessel Technology. 0773-78.

[5] Feng G H, Cao Y Z, Hao H. (2009) Research progress of heat transfer enhancement of shell-and-tube heat exchanger [J]. Technology \& Development of Chemical Industry. 0641-45.

[6] China National Standardization Management Committee. GB/T 151-2014, Heat exchanger [S].

[7] Xu C, Liu H H, Wang W J. (2009) The Research and Analysis of the Theoretical Calculation of Gas Explosion Limit and Its Influence Factors [J]. Shandong Coal Science and Technology. 4154-155.

[8] Zhao J K, Wang L, Hua S, Cao X. (2012) Numerical Simulation Study on Effect of Gas Concentration upon Gas Explosion [J]. Mining Safety \& Environmental Protection. 041-4+92.

[9] Zhang Z S, Yang C L. (2012) Application of Aspen Plus in chemical industry [J]. Guangdong Chemical Industry. 39 (4) 77-78.

[10] Anonymous. RealPennies.com: Turning Pennies into dollars: (OTC: AZPN) Aspen Technology, Inc [J]. M2 Presswire, 2009.

[11] Qian S W. (2002) Heat Exchanger Design Manual [M]. Chemical Industry Press, Beijing.

[12] Dang W Y, Yu A F. (2010) Study on the Design Pressure for Water-sealed Drum in Refinery Flare System [J]. China Safety Science Journal. 0975-80.

[13] Ma Z B. (2014) Research Status and Development Trend of Explosion Suppression Technology in Coal Mine [J]. Mining Safety \& Environmental Protection. 41 (2) 83-85.

[14] Xue S Q. (2010) Discussions on the development of automatic explosion suppression technology [J]. Mining Safety \& Environmental Protection. 0574-75.

[15] Huo C X. (2014) Analysis and Discussion on Explosion Accidents in Coal Mine Gas Pipeline [J]. Mining Safety \& Environmental Protection. 0191-94. 